\title{
ZNF804A genetic variation (rs1344706) affects brain grey but not white matter in schizophrenia and healthy subjects
}

\author{
I. Nenadic $^{1 *}$, R. Maitra ${ }^{1}$, F. B. Basmanav ${ }^{2}$, C. C. Schultz ${ }^{1}$, C. Lorenz ${ }^{1}$, C. Schachtzabel ${ }^{1}$, S. Smesny ${ }^{1}$, \\ M. M. Nöthen ${ }^{2,3}$, S. Cichon ${ }^{3,4,5}$, J. R. Reichenbach ${ }^{6}$, H. Sauer ${ }^{1}$, R. G. M. Schlösser ${ }^{1}$ and C. Gaser $^{1,7}$ \\ ${ }^{1}$ Department of Psychiatry and Psychotherapy, Jena University Hospital, Jena, Germany \\ ${ }^{2}$ Institute of Human Genetics, University of Bonn, Bonn, Germany \\ ${ }^{3}$ Department of Genomics, Life and Brain Center, University of Bonn, Bonn, Germany \\ ${ }^{4}$ Institute of Neuroscience and Medicine (INM-1), Research Center Jülich, Jülich, Germany \\ ${ }^{5}$ Division of Medical Genetics, Department of Biomedicine, University of Basel, Basel, Switzerland \\ ${ }^{6}$ Medical Physics Group, Institute for Diagnostic and Interventional Radiology I (IDIR I), Jena University Hospital, Germany \\ ${ }^{7}$ Department of Neurology, Jena University Hospital, Jena, Germany
}

Background. Genetic variation in the gene encoding ZNF804A, a risk gene for schizophrenia, has been shown to affect brain functional endophenotypes of the disorder, while studies of white matter structure have been inconclusive.

Method. We analysed effects of ZNF804A single nucleotide polymorphism rs1344706 on grey and white matter using voxel-based morphometry (VBM) in high-resolution T1-weighted magnetic resonance imaging scans of 62 schizophrenia patients and 54 matched healthy controls.

Results. We found a significant ( $p<0.05$, family-wise error corrected for multiple comparisons) interaction effect of diagnostic group $\mathrm{x}$ genotype for local grey matter in the left orbitofrontal and right and left lateral temporal cortices, where patients and controls showed diverging effects of genotype. Analysing the groups separately (at $p<0.001$, uncorrected), variation in rs1344706 showed effects on brain structure within the schizophrenia patients in several areas including the left and right inferior temporal, right supramarginal/superior temporal, right and left inferior frontal, left frontopolar, right and left dorsolateral/ventrolateral prefrontal cortices, and the right thalamus, as well as effects within the healthy controls in left lateral temporal, right anterior insula and left orbitofrontal cortical areas. We did not find effects of genotype of regional white matter in either of the two cohorts.

Conclusions. Our findings demonstrate effects of ZNF804A genetic variation on brain structure, with diverging regional effects in schizophrenia patients and healthy controls in frontal and temporal brain areas. These effects, however, might be dependent on the impact of other (genetic or non-genetic) disease factors.

Received 14 February 2014; Revised 25 April 2014; Accepted 25 April 2014; First published online 20 May 2014

Key words: Cerebral cortex, genetic variation, magnetic resonance imaging, psychosis, schizophrenia, voxel-based morphometry, ZNF804A.

\section{Introduction}

Schizophrenia has a substantial genetic basis, and a significant number of these genetic risk variants have recently been identified through genome-wide analyses (Sullivan et al. 2012). The mechanisms through which variation in risk genes, copy number variants, or rare mutations affect the developing brain and interact in its arising pathophysiology are still far from being clear. The ZNF804A gene (coding for zinc

\footnotetext{
* Address for correspondence: I. Nenadic, M.D., Department of Psychiatry and Psychotherapy, Jena University Hospital, Philosophenweg 3, 07743 Jena, Germany.

(Email: igor.nenadic@uni-jena.de)
}

finger protein $804 \mathrm{~A}$ ) has been suggested to harbour common risk variants for schizophrenia (O'Donovan et al. 2008), which has been replicated in several studies across different ethnic groups (Li et al. 2012; Zhang et al. 2012; Schwab et al. 2013). The rs1344706 single nucleotide polymorphism (SNP) has been of particular interest, as it has been linked to brain structural, functional and cognitive variation (Donohoe et al. 2010). Two recent studies have shown an impact of ZNF804A rs1344706 genetic variation on cognitive function and brain activation patterns in tasks related to cognitive control (Thurin et al. 2013) and working memory for faces (Linden et al. 2013), respectively, as well as connectivity measures in healthy subjects (Paulus et al. 2013). As such functions are putative 
endophenotypes for schizophrenia, the link to ZNF804A might provide an understanding on how the gene or gene products act on the biological substrates of psychosis, including schizophrenia and other psychiatric disorders (Donohoe et al. 2010; Hess \& Glatt, 2014).

ZNF804A might, however, also affect brain development; hence, brain structural changes related to variation in one or more SNPs may thus reflect an enduring impact of the gene's function (Donohoe et al. 2010). A first volumetric study of ZNF804A (rs1344706) in healthy subjects showed larger total white matter volumes in risk-allele carriers along with reduced grey matter in the angular gyrus, parahippocampal gyrus, posterior cingulate and medial orbitofrontal gyrus (Lencz et al. 2010). A subsequent volumetric study in schizophrenia patients and healthy controls demonstrated an effect of the same marker on total and frontal white matter volume in schizophrenia patients (risk-allele carriers having larger volumes), but only total white matter volume in healthy controls (Wassink et al. 2012). A more detailed recent voxelbased morphometry (VBM) study also revealed effects on regional grey matter in schizophrenia patients and healthy controls (Donohoe et al. 2011), whereby homozygous risk-allele carriers for rs1344706 differed in regional grey matter of the dorsolateral prefrontal cortex, hippocampus and amygdala compared with heterozygotes and non-risk-allele homozygotes. This last study, in particular, also suggested that effects of this polymorphism might, in fact, diverge markedly between healthy controls and schizophrenia patients, i.e. that effects vary both regionally and in direction, such as findings of increased hippocampal grey matter in risk-allele homozygotes in the patient sample, but not within the control sample (Donohoe et al. 2011). Similarly, we found cortical thickness to show dissociated effects in patients and controls (Schultz et al. 2014), with risk-allele carriers within the patient group having thicker prefrontal and temporal cortices, while in the healthy control group risk-allele homozygotes had thinner cortices. Despite the existing evidence, the effects and regional distribution remain unclear, and as with many other imaging genetics findings in schizophrenia, there is a lack of replication.

In the present study, we aimed to extend the evidence for effects of rs1344706 and its risk allele (A) on brain structure in both healthy controls and schizophrenia subjects by using VBM to map regional brain structural differences on a voxel-by-voxel basis, with the particular emphasis to replicate and extend the previous findings on $(a)$ the regional pattern of effects, and (b) the divergence of effects between schizophrenia patients and healthy controls.

\section{Method}

\section{Subjects}

We included 62 patients with a Diagnostic and Statistical Manual of Mental Disorders, fourth edition (DSM-IV) diagnosis of schizophrenia (44 male, 18 female; mean age 31.6 years, s.D. $=11.5$ years; all on stable antipsychotic medication) and 54 healthy controls from the community (29 male, 25 female; mean age 29.5 years, S.D. $=9.9$ years), who had no current or previous psychiatric treatment or diagnosis. All subjects provided written informed consent to a study protocol approved by the Jena Medical School Ethics Committee, in accordance with the Declaration of Helsinki. Groups did not differ in age ( $t$ test: $p=0.291$, two-tailed) or gender $\left(\chi^{2}\right.$ test: $\left.p=0.055\right)$. Groups differed in overall performance on the MWT-B (Mehrfachwahl-Wortschatz-Intelligenztest B), an estimate of pre-morbid intelligence quotient (IQ) [available for 58 schizophrenia patients and 37 controls; analysis of variance (ANOVA): $F=9.312, p=0.003$ ]. None of the subjects had any major medical or neurological condition. Part of this sample has been used in a recent analysis of ZNF804A effects on cortical thickness (Schultz et al. 2014), whereas for the present analysis, the sample has been expanded to allow analysis of a larger cohort with another methodological approach.

\section{Genotyping using Sanger sequencing}

Individual genotypes for the studied polymorphism were retrieved from sequence data generated by Sanger sequencing. Genomic DNA sequences covering rs1344706 and the flanking up- and downstream regions were retrieved from the UCSC Genome Browser based on the human genome build GRCh37/hg19 (http://genome.ucsc.edu/cgi-bin/hgGateway). Primer design was performed by keeping a minimum distance of about 150 base pairs (bp) up- and downstream from the target base. Primer sequences are obtainable from the authors upon request. Amplicons were generated under standard polymerase chain reaction (PCR) conditions. Resequencing was carried out with BigDye v3.1 (Applied Biosystems, USA) sequencing reagents employed according to the manufacturer's protocol. Data were generated on the Applied Biosystems 3130xl Genetic Analyzer, and SeqMan II (DNASTAR Inc., USA) software was used for data visualization. Electropherograms were evaluated independently by two raters.

Genotype distribution in schizophrenia patients was 23, 33 and six for AA, CA and CC, respectively, and 19, 28 and seven in healthy controls. Genotype distributions did not deviate significantly from HardyWeinberg equilibrium in patients and controls 
$\left(p_{\text {patients }}=0.234, p_{\text {controls }}=0.504\right)$. The rs1344706 minor (C) allele frequency (MAF) observed in our samples $(\mathrm{MAF}=0.375)$ was very similar to the MAF observed in 1000 Genomes (MAF=0.393; http://www.1000genomes. org/). ANOVA with the factors diagnosis and genotype did not show effects of diagnosis $(p=0.758)$, genotype $(p=0.188)$ or their interaction $(p=0.543)$ on the variable age. For pre-morbid IQ estimates (see above, available for 58 patients and 37 controls), there was also no effect of genotype ( $p=0.496)$, or diagnosis $\mathrm{x}$ genotype interaction $(p=0.114)$, but only for factor diagnosis (as shown above).

\section{Magnetic resonance imaging (MRI) and VBM}

We obtained T1-weighted high-resolution MRI scans on a $1.5 \mathrm{~T}$ Siemens Magnetom Vision plus system (Siemens, Germany) using a three-dimensional Fast Low Angle SHot (FLASH) sequence (repetition time= $15 \mathrm{~ms}$, echo time $=5 \mathrm{~ms}$, flip angle $\alpha=30^{\circ}, 192$ sagittal slices, field of view $=256 \times 256 \mathrm{~mm}$, with voxel dimensions $1 \times 1 \times 1 \mathrm{~mm}^{3}$ ). Images were visually inspected for artefacts and underwent automated quality control using an algorithm provided in the VBM toolbox (http://dbm.neuro.uni-jena.de/vbm8/).

For post-processing and VBM analysis, we used the VBM8 toolbox, a freely available toolbox based on Statistical Parametric Mapping software SPM8 (Institute of Neurology, UK) and Matlab (Mathworks, USA). VBM8 is based on the general VBM approach proposed by Ashburner \& Friston (2000, 2005), implementing the Diffeomorphic Anatomical Registration Through Exponentiated Lie Algebra (DARTEL) algorithm for non-linear normalization (Ashburner, 2007). Grey matter and white matter maps were extracted from MRI data for each subject and underwent nonlinear normalization using DARTEL. We defined an internal grey matter threshold of 0.2 for both grey matter maps and white matter maps, thus choosing a more conservative value to limit edge effects. Smoothing was performed with a $12 \mathrm{~mm}$ full-width at halfmaximum (FWHM) Gaussian kernel for grey matter maps and $20 \mathrm{~mm}$ FWHM for white matter maps. The Anatomical Automatic Labeling (AAL) toolbox was used for anatomical labelling (Tzourio-Mazoyer et al. 2002). In addition to regional analysis of grey and white matter, the VBM toolbox also estimates total brain grey matter and white matter volume by assigning the corresponding number of voxels to either tissue type multiplied by the voxel volumes, thus allowing analysis of effects of genotype on global parameters as well.

\section{Statistical analysis}

We first used multivariate ANOVA (with SPSS 20; IBM, USA) to test effects of genotype on total brain grey matter and total white matter volumes by defining the factors group (schizophrenia; controls) and genotype (AA; $\mathrm{CA}$; $\mathrm{CC}$ ) and the covariates age and gender (to remove variance related to these variables), and using Pillai's trace.

VBM statistics were carried out in SPM8 using a general linear model defining diagnostic group (schizophrenia; healthy control) and genotype (AA; CA; CC) while using age and gender as covariates (again, to remove their effect on variance) and applying a height threshold of $p<0.001$ (uncorrected) throughout all analyses. This choice of threshold was based on the existence of previous VBM analyses, as mentioned above, and thus allowed us to either replicate (or refute) previous findings in these mentioned areas, while whole-brain testing allowed us to expand analysis to other areas as well. Our main hypothesis of divergent effects of rs1344706 was tested with an interaction of group $\mathrm{x}$ genotype; furthermore, we also tested effects of genotype in schizophrenia patients and effects of genotype in healthy controls separately; we performed each of these three tests for both grey and white matter, respectively.

\section{Results}

\section{Total brain grey matter and white matter}

Overall multivariate ANOVA (for grey matter and white matter) revealed no significant effect of factor diagnosis $\left(F_{2,107}=0.223, p=0.801\right)$ or genotype $\left(F_{4,216}=\right.$ $1.831, p=0.124)$, but a significant diagnosis $\mathrm{x}$ genotype interaction $\left(F_{4,216}=3.109, p=0.016\right)$. Tests of betweensubject effects revealed significant group differences only for the interaction of diagnosis $x$ genotype for total brain white matter $\left(F_{2,116}=3.318, p=0.04\right)$, but not for grey matter $\left(F_{2,216}=1.267, p=0.286\right)$ and neither for grey nor white matter for the diagnosis or genotype effects alone. Both age and gender had significant effects on total brain grey matter and white matter, respectively (both $p<0.001$ ).

\section{VBM analysis of grey matter}

In schizophrenia patients, there were several significant regional effects of genotype on grey matter (see Fig. 1), including the left temporal pole and anterior part of the inferior temporal gyrus, bilateral lateral temporal cortices, right superior temporal and supramarginal gyri, bilateral inferior prefrontal gyri, left medial cerebellum, left orbitofrontal cortex and posterior right thalamus.

In healthy controls, we found several significant regional effects of genotype (see Fig. 2), including the left middle temporal gyrus (lateral temporal cortex), 


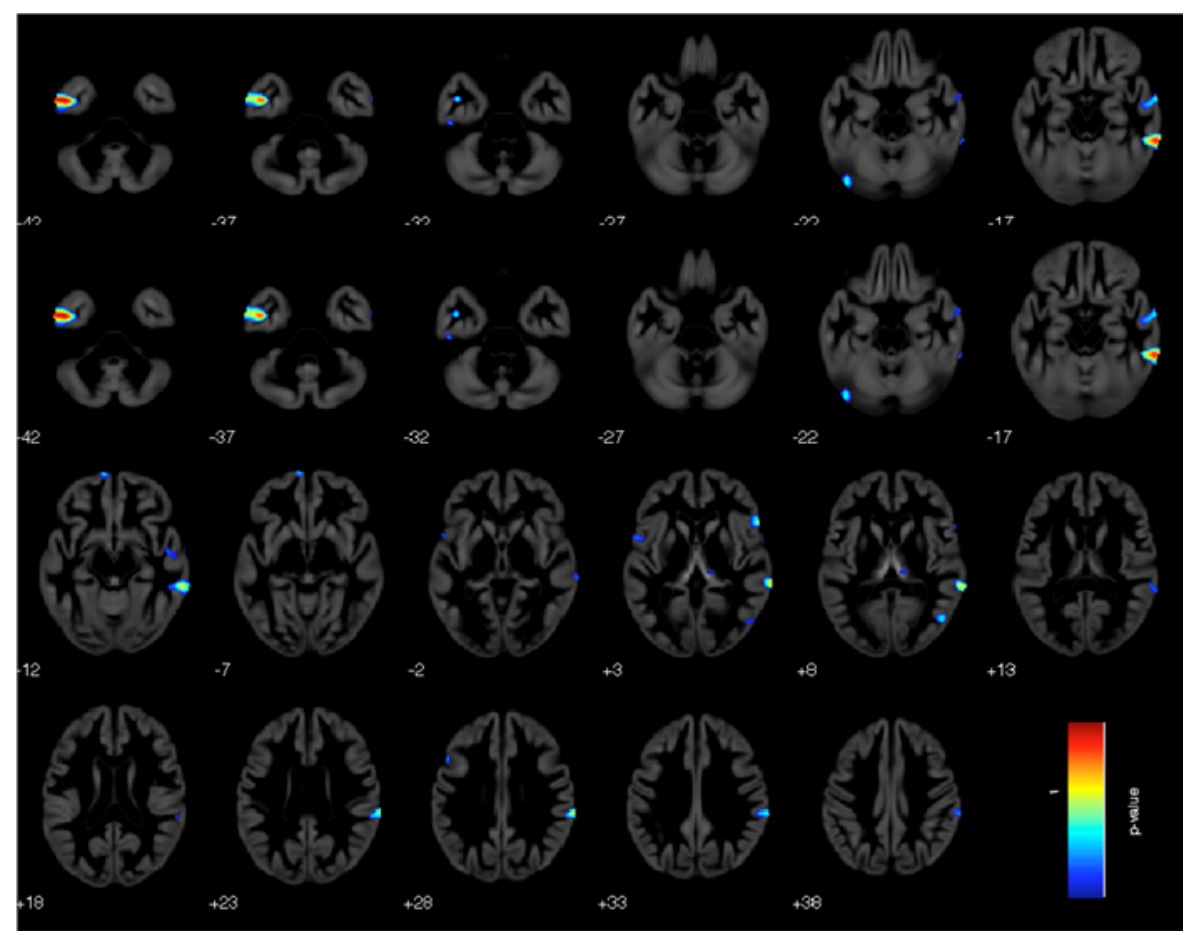

Fig. 1. Voxel-based morphometry analysis of effects of ZNF804A genotype (rs1344706) on grey matter in schizophrenia patients $(p<0.001$, uncorrected).

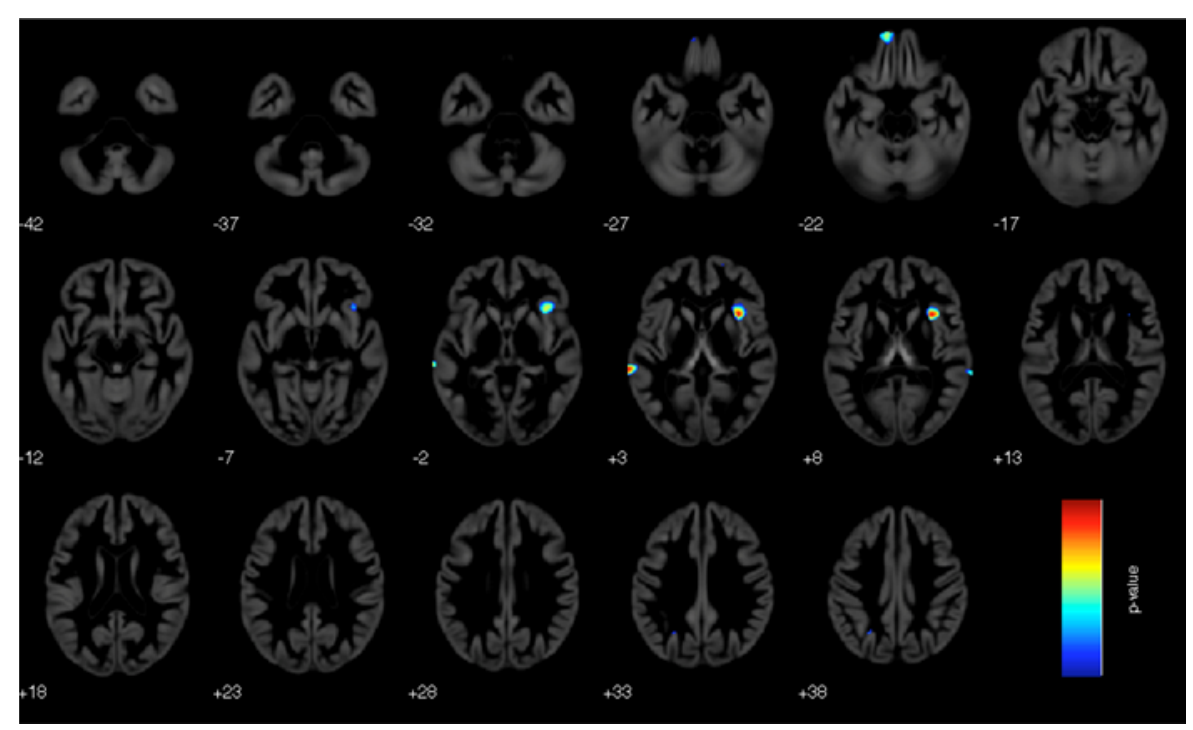

Fig. 2. Voxel-based morphometry analysis of effects of ZNF804A genotype (rs1344706) on grey matter in healthy controls $(p<0.001$, uncorrected $)$.

right insular cortex, left orbitofrontal cortex and right superior temporal cortex.

The interaction analysis of diagnosis $\mathrm{x}$ genotype for regional grey matter also resulted in significant findings (see Fig. 3) in some of the above-mentioned clusters. Of note, parts of three of these clusters actually showed significance levels that also survived family-wise error (FWE) correction for multiple comparisons (as implemented in SPM, peak-level): one cluster in the left orbitofrontal cortex (coordinates $-6,62,-20$; FWE-corrected $p=0.021)$, right middle/ superior temporal gyrus $(69,-39,6$; FWE-corrected $p=0.029)$ and left middle temporal gyrus $(-68,-39$, 3; FWE-corrected $p=0.034$ ) (see Table 1 and Fig. 4). 


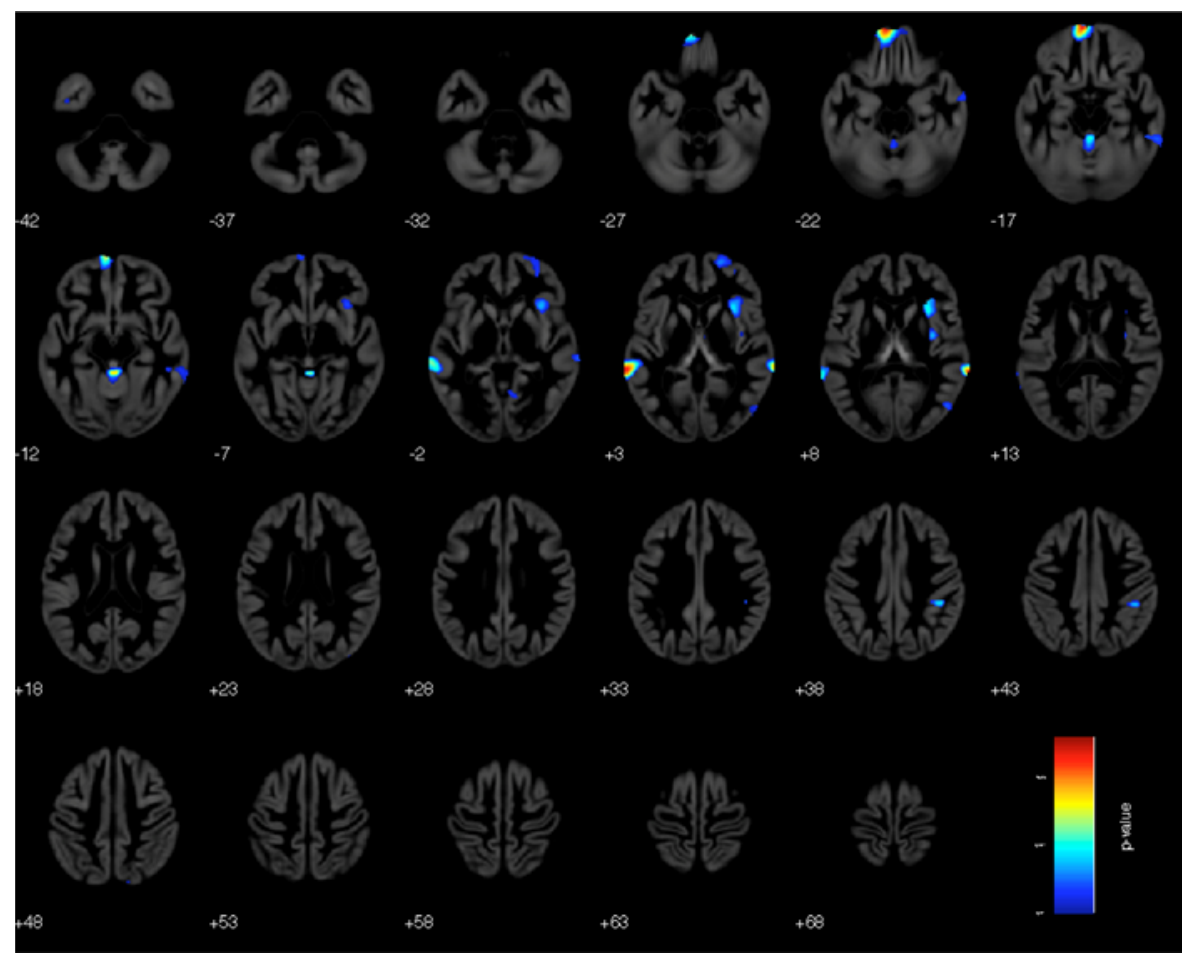

Fig. 3. Voxel-based morphometry analysis of interaction effects of ZNF804A genotype (rs1344706) x diagnostic group (schizophrenia; healthy controls) $(p<0.001$, uncorrected).

Results of group comparison of grey matter differences between patients and healthy controls are provided in online Supplementary Fig. S1.

In addition to the above results, we inspected the plots for profiles of genotype-related changes, i.e. to identify the subgroup contributing most to the effects. Assessing the distribution of grey matter values across the groups, a rather consistent pattern emerged. For the interaction analysis, the effect was caused by smaller grey matter values for AA homozygotes (compared with AC heterozygotes) in the healthy control group, whereas in the schizophrenia group grey matter values for AA homozygotes were higher compared with AC heterozygotes. The CC homozygotes often took an intermediary position. However, these subgroups were very small, thus making direct comparison more difficult. For the genotype effects within each group, we found that AA homozygotes in the schizophrenia group had higher grey matter values than the AC heterozygotes across all significant larger clusters. Within the healthy control group analysis, inspection of plots also confirmed smaller grey matter values for AA homozygotes (in significant clusters) in contrast to AC heterozygotes (except for the anterior insula cluster, where the two groups were similar, but CC homozygotes showed larger effects), thus confirming the overall pattern of an inverted (or diverging) effect of AA versus AC carrier status in schizophrenia patients versus controls.

\section{VBM analysis of white matter}

There were no significant effects of genotype on white matter in either schizophrenia patients or healthy controls, and no significant effects of a diagnosis $x$ genotype interaction for white matter (all at the predefined threshold of $p<0.001$ ).

\section{Discussion}

As its main result, our study identified effects of ZNF804A on regional brain structure in the left orbitofrontal and left and right lateral temporal cortices with a significant diagnostic group $\mathrm{x}$ genotype interaction. In these regions, variation in rs1344706 led to diverging effects in patients and controls. Further major effects (at uncorrected levels of significance) were seen in multiple frontal, temporal and parietal cortical areas, where the gene's impact was different for the two diagnostic groups. While these findings add to the increasing identification of brain structural effects of this schizophrenia risk gene, they partially confirm a peculiar effect of this SNP. This effect is related to the markedly different impact of the SNP in patients and healthy subjects. Previous morphometry studies have already suggested such effects on grey matter density (Donohoe et al. 2011), as well as on cortical thickness in our own previous work (Schultz et al. 2014). There is also increasing evidence for ZNF804A effects 
Table 1. Anatomical overview of regions with significant effects ( $p<0.001$, uncorrected) in voxel-based morphometry analysis of ZNF804A ( $r$ 1344706) genotype effects (only clusters with at least $k=10$ voxels listed) in schizophrenia patients, healthy controls, and interaction effects of diagnosis $x$ genotype

\begin{tabular}{|c|c|c|}
\hline Anatomical region & $\begin{array}{l}\text { MNI coordinates of } \\
\text { maximum voxel: } x, y, z\end{array}$ & $\begin{array}{l}k \text {, number } \\
\text { of voxels }\end{array}$ \\
\hline \multicolumn{3}{|l|}{ Genotype effects in schizophrenia patients $(p<0.001)$} \\
\hline Left inferior temporal cortex & $-50,-3,-45$ & 1180 \\
\hline Right inferior (/middle) temporal cortex & $64,-40,-17$ & 664 \\
\hline Right supramarginal gyrus, superior temporal cortex & $69,-37,4$ & 907 \\
\hline Right inferior frontal gyrus & $58,21,3$ & 252 \\
\hline Left cerebellum & $-42,-78,-23$ & 151 \\
\hline Left medial orbitofrontal cortex & $-8,66,-11$ & 120 \\
\hline Right middle temporal gyrus & $66,-1,-17$ & 438 \\
\hline Right middle temporal/middle occipital cortex & $48,-69,7$ & 181 \\
\hline Left lower cerebellum & $-14,-81,-50$ & 101 \\
\hline Left inferior frontal gyrus & $-51,15,28$ & 31 \\
\hline Left rolandic operculum/temporal pole & $-56,8,0$ & 120 \\
\hline Left inferior temporal cortex & $-48,-25,-33$ & 79 \\
\hline Right inferior temporal cortex & $58,-1,-39$ & 33 \\
\hline Right temporal pole/superior temporal gyrus & $62,3,1$ & 12 \\
\hline Right thalamus & $12,-25,6$ & 70 \\
\hline \multicolumn{3}{|l|}{ Genotype effects in healthy controls $(p<0.001)$} \\
\hline Left middle temporal gyrus & $-68,-34,1$ & 181 \\
\hline Right insula & $33,15,6$ & 770 \\
\hline Left superior orbitofrontal cortex/gyrus rectus & $-9,57,-23$ & 233 \\
\hline Right superior temporal gyrus & $69,-39,9$ & 45 \\
\hline Left middle occipital cortex & $-24,-60,36$ & 42 \\
\hline \multicolumn{3}{|l|}{ Interaction effects of diagnosis $\mathrm{x}$ genotype $(p<0.001)$} \\
\hline Left gyrus rectus/orbitofrontal cortex & $-6,62,-10^{*}$ & $1291^{*}$ \\
\hline Right middle/superior temporal gyrus & $69,-39,6^{*}$ & $371^{*}$ \\
\hline Left middle temporal gyrus & $-68,-39,3^{*}$ & $834^{*}$ \\
\hline Vermis/left cerebellum & $2,-40,-12$ & 651 \\
\hline Right supramarginal gyrus & $42,-37,37$ & 217 \\
\hline Right insula & $32,17,7$ & 950 \\
\hline Right inferior/middle temporal gyrus & $52,-39,-15$ & 364 \\
\hline Right insula & $34,-7,9$ & 199 \\
\hline Right superior/middle frontal gyrus & $20,60,3$ & 535 \\
\hline Right middle temporal/occipital cortex & $50,-72,7$ & 160 \\
\hline Right middle temporal gyrus & $63,-4,-21$ & 119 \\
\hline Right lingual gyrus & $9,-61,-3$ & 76 \\
\hline Left inferior temporal cortex & $-42,-6,-42$ & 20 \\
\hline Left middle occipital cortex & $-38,-78,3$ & 11 \\
\hline
\end{tabular}

MNI, Montreal Neurological Institute.

* Parts of these clusters are also significant at $p<0.05$, family-wise error corrected.

on brain function. Two recent electroencephalography (EEG) studies revealed an impact of rs1344706 on P300 amplitude/responses (Del Re et al. 2014; O'Donoghue et al. 2014).

Imaging genetics studies have generally aimed to evaluate the effect of particular risk genes (or risk scores) on brain structures. Often, however, it is unclear how gene expression or gene products might influence brain structure in later life. For ZNF804A, there is growing evidence that effects start during early brain development. This has been shown in fetal brains, where rs1344706 variation had a strong impact on ZNF804A allelic expression during the second trimester of intra-uterine development, but not in adult brains (Hill \& Bray, 2012). Expression in postmortem brains seems to differ between patients and healthy controls, but this observation is only incompletely understood (Okada et al. 2012; 


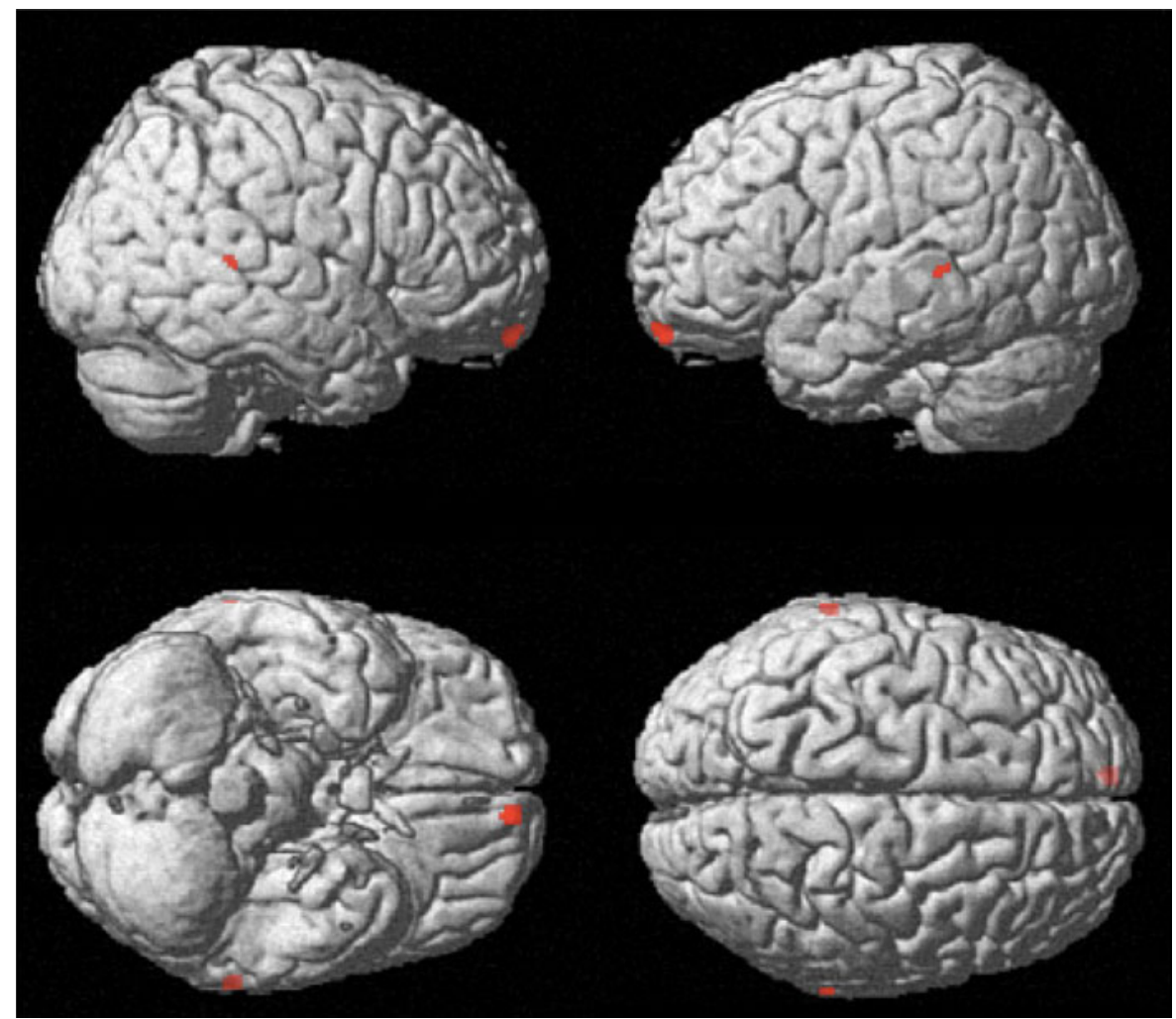

Fig. 4. Render view of voxel-based morphometry analysis of interaction effects of ZNF804A genotype (rs1344706) $x$ diagnostic group (schizophrenia; healthy controls) ( $p<0.05$, family-wise error correction for multiple comparisons).

Schultz et al. 2014), as is the regional pattern of physiological expression (Buonocore et al. 2010). Also, recent studies suggest an indirect action of ZNF804A by modulating the gene expression of other risk genes (Girgenti et al. 2012).

While in many instances genetic risk factors have demonstrated stronger impact of a particular risk gene in patients compared with controls, this might not be the case (at least not generally) for rs1344706. While the mentioned VBM study of Donohoe et al. (2011) reported preserved brain structure in patients, one volumetric study found larger regional white matter volumes in risk-allele carriers, together, however, with more severe psychotic symptoms (Wassink et al. 2012). In contrast, a large study of healthy subjects did not find statistically significant effects on brain structure (Cousijn et al. 2012). Given that volumetry, VBM and analysis of cortical thickness assess three different aspects of brain morphometry, our findings are most comparable with the studies of Donohoe et al. (2011) with respect to methods. In fact, these authors analysed schizophrenia and healthy control cohorts of roughly similar size to ours (using the older VBM5 toolbox). While their regional findings only partially overlap with ours (including anterior temporal pole and temporal cortical areas, although with somewhat different location), they also share similarities. The authors reported that AA risk homozygotes showed opposite effects in patient versus control groups, with higher grey matter density for AA homozygotes in patients, but not controls. Similarly, effects of ZNF804A on cortical thickness have received support from two recent studies (Voineskos et al. 2011; Schultz et al. 2014), though there has been also one failure to replicate (Bergmann et al. 2013).

Functional studies, however, do suggest effects of ZNF804A genotypes in patients to exceed those seen in healthy controls (Rasetti et al. 2011). Given that there are also a number of studies demonstrating effects of rs1344706 in healthy subjects (Esslinger et al. 2009; Walter et al. 2011; Linden et al. 2013; Mohnke et al. 2014), further studies might elucidate (within the same cohort) the interaction of structural and functional effects.

Our study failed to identify any affects of ZNF804A on white matter density. Although VBM of white matter is inferior to diffusion tensor imaging (DTI) applications, which captures also quantitative measures of white matter damage (e.g. assessing fractional anisotropy), our results fit into the current literature on ZNF804A and white matter tracts. Previous DTI studies have mostly failed to confirm a clear impact of ZNF804A on white matter architecture or fractional anisotropy measures (Voineskos et al. 2011; Sprooten 
et al. 2012; Wei et al. 2013). One previous white matter VBM study (of T1-weighted MRI) suggested an interaction of group and genotype (rs1344706) in left prefrontal white matter (Wei et al. 2012), but our study did not replicate this finding. In two more recent studies, one found lower fractional anisotropy in risk-allele carriers in several regions including the corpus callosum and left forceps minor (Ikuta et al. 2014), while a second study failed to show any significant impact of ZNF804A on white matter microstructure in a large healthy subject sample ( $\mathrm{O}^{\prime}$ Donoghue et al. 2014).

\section{Conclusions}

In conclusion, our study provides evidence for significant divergence of ZNF804A (rs1344706) effects on grey matter in prefrontal and temporal brain areas. Effects of prefrontal, temporal and parietal grey matter differ not only according to genotype, but the genotype-related effects are different between schizophrenia patients and controls. There was, however, no effect on white matter density. While our results confirm divergence of structural effects across different groups, further studies are needed to clarify the microstructural changes underlying these effects.

\section{Supplementary material}

For supplementary material accompanying this paper visit http://dx.doi.org/10.1017/S0033291714001159.

\section{Acknowledgements}

I.N. was supported by a Junior Scientist Grant from IZKF (Interdisiplinäres Zentrum für klinische Forschung, Jena). The work of M.M.N. and S.C. was supported by the German Federal Ministry of Education and Research (BMBF) through the Integrated Genome Research Network (IG) MooDS (Systematic Investigation of the Molecular Causes of Major Mood Disorders and Schizophrenia), under the auspices of the National Genome Research Network plus (NGFNplus) and through the Integrated Network IntegraMent (Integrated Understanding of Causes and Mechanisms in Mental Disorders), under the auspices of the e:Med Programme. M.M.N. is a member of the German Research Foundation (DFG)funded Excellence-Cluster ImmunoSensation. He also received support from the Alfried Krupp von Bohlen und Halbach-Stiftung.

\section{Declaration of Interest}

None.

\section{References}

Ashburner J (2007). A fast diffeomorphic image registration algorithm. Neuroimage 38, 95-113.

Ashburner J, Friston KJ (2000). Voxel-based morphometrythe methods. Neuroimage 11, 805-821.

Ashburner J, Friston KJ (2005). Unified segmentation. Neuroimage 26, 839-851.

Bergmann O, Haukvik UK, Brown AA, Rimol LM, Hartberg CB, Athanasiu L, Melle I, Djurovic S, Andreassen OA, Dale AM, Agartz I (2013). ZNF804A and cortical thickness in schizophrenia and bipolar disorder. Psychiatry Research 212, 154-157.

Buonocore F, Hill MJ, Campbell CD, Oladimeji PB, Jeffries AR, Troakes C, Hortobagyi T, Williams BP, Cooper JD, Bray NJ (2010). Effects of cis-regulatory variation differ across regions of the adult human brain. Human Molecular Genetics 19, 4490-4496.

Cousijn H, Rijpkema M, Harteveld A, Harrison PJ, Fernandez G, Franke B, Arias-Vasquez A (2012). Schizophrenia risk gene ZNF804A does not influence macroscopic brain structure: an MRI study in 892 volunteers. Molecular Psychiatry 17, 1155-1157.

Del Re EC, Bergen SE, Mesholam-Gately RI, Niznikiewicz MA, Goldstein JM, Woo TU, Shenton ME, Seidman LJ, McCarley RW, Petryshen TL (2014). Analysis of schizophrenia-related genes and electrophysiological measures reveals ZNF804A association with amplitude of P300b elicited by novel sounds. Translational Psychiatry 4, e346.

Donohoe G, Morris DW, Corvin A (2010). The psychosis susceptibility gene ZNF804A: associations, functions, and phenotypes. Schizophrenia Bulletin 36, 904-909.

Donohoe G, Rose E, Frodl T, Morris D, Spoletini I, Adriano F, Bernardini S, Caltagirone C, Bossu P, Gill M, Corvin AP, Spalletta G (2011). ZNF804A risk allele is associated with relatively intact gray matter volume in patients with schizophrenia. Neuroimage 54, 2132-2137.

Esslinger C, Walter H, Kirsch P, Erk S, Schnell K, Arnold C, Haddad L, Mier D, Opitz von Boberfeld C, Raab K, Witt SH, Rietschel M, Cichon S, Meyer-Lindenberg A (2009). Neural mechanisms of a genome-wide supported psychosis variant. Science 324, 605.

Girgenti MJ, LoTurco JJ, Maher BJ (2012). ZNF804a regulates expression of the schizophrenia-associated genes PRSS16, COMT, PDE4B, and DRD2. PLOS ONE 7, e32404.

Hess JL, Glatt SJ (2014). How might ZNF804A variants influence risk for schizophrenia and bipolar disorder? A literature review, synthesis, and bioinformatic analysis. American Journal of Medical Genetics B: Neuropsychiatric Genetics 165, 28-40.

Hill MJ, Bray NJ (2012). Evidence that schizophrenia risk variation in the ZNF804A gene exerts its effects during fetal brain development. American Journal of Psychiatry 169, 1301-1308.

Ikuta T, Peters BD, Guha S, John M, Karlsgodt KH, Lencz T, Szeszko PR, Malhotra AK (2014). A schizophrenia risk gene, ZNF804A, is associated with brain white matter microstructure. Schizophrenia Research 155, 15-20. 
Lencz T, Szeszko PR, DeRosse P, Burdick KE, Bromet EJ, Bilder RM, Malhotra AK (2010). A schizophrenia risk gene, ZNF804A, influences neuroanatomical and neurocognitive phenotypes. Neuropsychopharmacology 35, 2284-2291.

Li M, Shi CJ, Shi YY, Luo XJ, Zheng XB, Li ZQ, Liu JJ, Chong SA, Lee J, Wang Y, Liu XY, Yin LD, Pu XF, Diao HB, Xu Q, Su B (2012). ZNF804A and schizophrenia susceptibility in Asian populations. American Journal of Medical Genetics B: Neuropsychiatric Genetics 159B, 794-802.

Linden DE, Lancaster TM, Wolf C, Baird A, Jackson MC, Johnston SJ, Donev R, Thome J (2013). ZNF804A genotype modulates neural activity during working memory for faces. Neuropsychobiology 67, 84-92.

Mohnke S, Erk S, Schnell K, Schutz C, Romanczuk-Seiferth N, Grimm O, Haddad L, Pohland L, Garbusow M, Schmitgen MM, Kirsch P, Esslinger C, Rietschel M, Witt SH, Nothen MM, Cichon S, Mattheisen M, Muhleisen T, Jensen J, Schott BH, Maier W, Heinz A, Meyer-Lindenberg A, Walter $\mathbf{H}$ (2014). Further evidence for the impact of a genomewide-supported psychosis risk variant in ZNF804A on the theory of mind network. Neuropsychopharmacology 39, 1196-1205.

O'Donoghue T, Morris DW, Fahey C, Da Costa A, Moore S, Cummings E, Leicht G, Karch S, Hoerold D, Tropea D, Foxe JJ, Gill M, Corvin A, Donohoe G (2014). Effects of ZNF804A on auditory P300 response in schizophrenia. Translational Psychiatry 4, e345.

O'Donovan MC, Craddock N, Norton N, Williams H, Peirce T, Moskvina V, Nikolov I, Hamshere M, Carroll L, Georgieva L, Dwyer S, Holmans P, Marchini JL, Spencer CC, Howie B, Leung HT, Hartmann AM, Möller HJ, Morris DW, Shi Y, Feng G, Hoffmann P, Propping P, Vasilescu C, Maier W, Rietschel M, Zammit S, Schumacher J, Quinn EM, Schulze TG, Williams NM, Giegling I, Iwata N, Ikeda M, Darvasi A, Shifman S, He L, Duan J, Sanders AR, Levinson DF, Gejman PV, Cichon S, Nothen MM, Gill M, Corvin A, Rujescu D, Kirov G, Owen MJ, Buccola NG, Mowry BJ, Freedman R, Amin F, Black DW, Silverman JM, Byerley WF, Cloninger CR; Molecular Genetics of Schizophrenia Collaboration (2008). Identification of loci associated with schizophrenia by genome-wide association and follow-up. Nature Genetics 40, 1053-1055.

Okada T, Hashimoto R, Yamamori H, Umeda-Yano S, Yasuda Y, Ohi K, Fukumoto M, Ikemoto K, Kunii Y, Tomita H, Ito A, Takeda M (2012). Expression analysis of a novel mRNA variant of the schizophrenia risk gene ZNF804A. Schizophrenia Research 141, 277-278.

Paulus FM, Krach S, Bedenbender J, Pyka M, Sommer J, Krug A, Knake S, Nothen MM, Witt SH, Rietschel M, Kircher T, Jansen A (2013). Partial support for ZNF804A genotype-dependent alterations in prefrontal connectivity. Human Brain Mapping 34, 304-313.

Rasetti R, Sambataro F, Chen Q, Callicott JH, Mattay VS, Weinberger DR (2011). Altered cortical network dynamics: a potential intermediate phenotype for schizophrenia and association with ZNF804A. Archives of General Psychiatry 68, 1207-1217.

Schultz CC, Nenadic I, Riley B, Vladimirov VI, Wagner G, Koch K, Schachtzabel C, Muhleisen TW, Basmanav B, Nothen MM, Deufel T, Kiehntopf M, Rietschel M, Reichenbach JR, Cichon S, Schlosser RG, Sauer H (2014). ZNF804A and cortical structure in schizophrenia: in vivo and postmortem studies. Schizophrenia Bulletin 40, 532-541.

Schwab SG, Kusumawardhani AA, Dai N, Qin W, Wildenauer MD, Agiananda F, Amir N, Antoni R, Arsianti T, Asmarahadi A, Diatri H, Djatmiko P, Irmansyah I, Khalimah S, Kusumadewi I, Kusumaningrum P, Lukman PR, Mustar L, Nasrun MW, Naswati S, Prasetiyawan P, Semen GM, Siste K, Tobing H, Widiasih N, Wiguna T, Wulandari WD; Indonesian Schizophrenia Genetics Consortium, Benyamin B, Wildenauer DB (2013). Association of rs1344706 in the ZNF804A gene with schizophrenia in a case/control sample from Indonesia. Schizophrenia Research 147, 46-52.

Sprooten E, McIntosh AM, Lawrie SM, Hall J, Sussmann JE, Dahmen N, Konrad A, Bastin ME, Winterer G (2012). An investigation of a genomewide supported psychosis variant in ZNF804A and white matter integrity in the human brain. Magnetic Resonance Imaging 30, 1373-1380.

Sullivan PF, Daly MJ, O'Donovan M (2012). Genetic architectures of psychiatric disorders: the emerging picture and its implications. Nature Review Genetics 13, 537-551.

Thurin K, Rasetti R, Sambataro F, Safrin M, Chen Q, Callicott JH, Mattay VS, Weinberger DR (2013). Effects of ZNF804A on neurophysiologic measures of cognitive control. Molecular Psychiatry 18, 852-854.

Tzourio-Mazoyer N, Landeau B, Papathanassiou D, Crivello F, Etard O, Delcroix N, Mazoyer B, Joliot M (2002). Automated anatomical labeling of activations in SPM using a macroscopic anatomical parcellation of the MNI MRI single-subject brain. Neuroimage 15, 273-289.

Voineskos AN, Lerch JP, Felsky D, Tiwari A, Rajji TK, Miranda D, Lobaugh NJ, Pollock BG, Mulsant BH, Kennedy JL (2011). The ZNF804A gene: characterization of a novel neural risk mechanism for the major psychoses. Neuropsychopharmacology 36, 1871-1878.

Walter H, Schnell K, Erk S, Arnold C, Kirsch P, Esslinger C, Mier D, Schmitgen MM, Rietschel M, Witt SH, Nothen MM, Cichon S, Meyer-Lindenberg A (2011). Effects of a genome-wide supported psychosis risk variant on neural activation during a theory-of-mind task. Molecular Psychiatry 16, 462-470.

Wassink TH, Epping EA, Rudd D, Axelsen M, Ziebell S, Fleming FW, Monson E, Ho BC, Andreasen NC (2012). Influence of ZNF804a on brain structure volumes and symptom severity in individuals with schizophrenia. Archives of General Psychiatry 69, 885-892.

Wei Q, Kang Z, Diao F, Guidon A, Wu X, Zheng L, Li L, Guo X, Hu M, Zhang J, Liu C, Zhao J (2013). No association of ZNF804A rs1344706 with white matter integrity in schizophrenia: a tract-based spatial statistics study. Neuroscience Letters 532, 64-69. 
Wei Q, Kang Z, Diao F, Shan B, Li L, Zheng L, Guo X, Liu C, Zhang J, Zhao J (2012). Association of the ZNF804A gene polymorphism rs1344706 with white matter density changes in Chinese schizophrenia. Progress in Neuropsychopharmacology and Biological Psychiatry 36, 122-127.
Zhang R, Yan JD, Valenzuela RK, Lu SM, Du XY, Zhong B, Ren J, Zhao SH, Gao CG, Wang L, Guo TW, Ma J (2012). Further evidence for the association of genetic variants of ZNF804A with schizophrenia and a meta-analysis for genome-wide significance variant rs1344706. Schizophrenia Research 141, 40-47. 